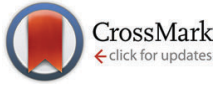

Cite this: Mater. Chem. Front., 2017, 1, 152

Received 25th May 2016,

Accepted 29th June 2016

DOI: $10.1039 / c 6 q m 00065 g$

rsc.li/frontiers-materials

\title{
Thiophene-derived polymer dots for imaging endocytic compartments in live cells and broad-spectrum bacterial killing $\dagger$
}

\author{
Kenath Priyanka Prasad, ${ }^{a}$ Aung Than, ${ }^{a}$ Nan Li, ${ }^{a}$ Mahasin Alam SK, ${ }^{a}$ Hongwei Duan, ${ }^{a}$ \\ Kanyi Pu, ${ }^{a}$ Xinting Zheng ${ }^{b}$ and Peng Chen ${ }^{\star a}$ \\ In this work, we present a new type of polymer dot synthesized from bithiophene (pTh-Pdot) via a \\ simple and high yield procedure. Owing to their high brightness, excellent photo-stability, good \\ biocompatibility, and molecular size, pTh-Pdots are promising for bioimaging applications. As a proof- \\ of-concept demonstration, pTh-Pdots are herein employed to track the endocytic pathway in live cells. \\ Furthermore, we show that these polymeric nanoparticles can serve as potent antibacterial agents by \\ making use of their ability to disrupt bacterial membranes and high peroxidase mimicking activity.
}

\section{Introduction}

In recent years, fluorescent quantum dots or nanoparticles derived from semiconducting polymers (Pdots) have attracted considerable interest for various biomedical applications (e.g., fluorescence and photoacoustic cellular imaging, optical sensing, and photodynamic therapy), owing to their unique properties such as high brightness, tunable and non-blinking emission, broad absorption, high photo-stability, biocompatibility, and molecular size. ${ }^{1-6}$ Pdots are usually made by collapsing and packing long-chain fluorescent polymers using, e.g., miniemulsion or reprecipitation methods. ${ }^{7-9}$ These Pdots are often large in size and require a tedious synthesis process.

Recently, we synthesized a new type of crystalline Pdot by ultrasonic breakdown of electrochemically polymerized nonfluorescent poly(3,4-ethylenedioxythiophene). ${ }^{10}$ Using a similar strategy, we herein fabricate novel Pdots from polythiophene, a semiconducting polymer. These readily synthesized Pdots are of molecular size, bright, and biocompatible. Interestingly, such polythiophene-based Pdots (pTh-Pdots) exhibit negative solvatochromism, i.e., a blue shift in photoluminescence (PL) emission with an increase in solvent polarity. We demonstrate that, likely due to its negative solvatochromism properties, pTh-Pdots fluoresce blue in early endosomes but green in lysosomes, hence allowing tracking of the endocytic pathway

\footnotetext{
${ }^{a}$ School of Chemical and Biomedical Engineering, Nanyang Technological University, 70 Nanyang Drive, 637457, Singapore.E-mail: ChenPeng@ntu.edu.sg ${ }^{b}$ Institute of Materials Research and Engineering, the Agency for Science, Technology and Research (A*STAR), 2 Fusionopolis Way, \#08-03, Innovis, 138634, Singapore

$\dagger$ Electronic supplementary information (ESI) available: Characterization, cell imaging and antibacterial studies. See DOI: 10.1039/c6qm00065g
}

in live cells. In addition to their potential for cellular imaging, we further show the use of pTh-Pdots as an excellent antibacterial agent against both Gram-positive and Gram-negative bacteria.

\section{Experimental section}

Preparation of polythiophene (pTh) films

The 2,2'-bithiophene (BTh) monomer and ionic liquids (1- $n$-butyl3-methylimidazolium tetrafluoroborate - $\mathrm{BMIMBF}_{4}$; 1-butyl-3methylimidazolium methyl sulphate - $\mathrm{BMIMMeSO}_{4}$ ) were purchased from Sigma Aldrich.

Using a $0.1 \mathrm{M}$ BTh monomer in the ionic liquid BMIMBF 4 , the poly(2,2-bithiophene) (pTh) film was electro-polymerized onto an ITO working electrode held at the constant potential of $5 \mathrm{~V}$ for 45 minutes. Electro-polymerization was done using a standard three-electrode configuration consisting of a platinum plate (counter) electrode, a silver wire (reference) electrode and an indium tin oxide (ITO) (working) electrode on an electrochemical workstation (CHI 660D). Overnight drying in a $37{ }^{\circ} \mathrm{C}$ vacuum oven was done after extensive post-polymerization washing to eliminate excess ionic liquid and unreacted monomers. Polymer films using different ionic liquids such as $\mathrm{BMIMMeSO}_{4}$ were prepared for optimization studies.

\section{Preparation of polymer dots (Pdots)}

The dried pTh polymer film was gently scraped off the ITO electrode and suspended in THF (tetrahydrofuran, Sigma Aldrich) solvent at $1 \mathrm{mg} \mathrm{m} \mathrm{ml}^{-1}$ concentration, followed by exfoliation using ultrasonication (Branson 2510; $1.1 \mathrm{~A}, 230 \mathrm{~W}$ ) at $27{ }^{\circ} \mathrm{C}$ for $1 \mathrm{~h}$. The suspension formed was then filtered using a WHATMAN $0.2 \mu \mathrm{m}$ PTFE filter to obtain a clear yellow 
solution. Eventually, the THF was extracted using a rotary evaporator (Heidolf) at $30{ }^{\circ} \mathrm{C}$.

The dried aggregates were subsequently re-suspended in DMF ( $N, N$-dimethylformide, Sigma Aldrich) or distilled water following which the polythiophene-based Pdot (pTh-Pdots) suspensions were further purified. pTh-Pdots in DMF (green pTh-Pdots) were purified by syringe filtration through a $0.1 \mu \mathrm{m}$ PTFE filter. On the other hand, the pTh-Pdots in water (blue pTh-Pdots) were ultra-filtered (molecular cut-off weight of $3 \mathrm{kDa}$ ) followed by filtration through a $0.1 \mu \mathrm{m}$ nitrocellulose membrane to remove the aggregates. Both the green pTh-Pdots and blue pTh-Pdots were highly stable (lasting for months without apparent aggregation). Furthermore, aprotic solvents such as THF appeared to be suitable for high yield exfoliations in contrast to other solvents like ethanol and water which showed little to no exfoliation (data not shown).

\section{Characterization}

The samples were imaged using a field emission scanning electron microscope (FESEM, JMS-6700F), an atomic force microscope (MFP-3D AFM microscope, Asylum research), and a high-resolution transmission electron microscope (HRTEM, JEOL JEM 2100F TEM). Fourier transform infrared spectroscopy and X-ray photoelectron spectroscopy were performed using a Perkin Elmer FTIR Spectrometer (GX 69233), and a Kratos Axis Ultra ${ }^{\text {DLD }}$ spectrometer (Kratos Analytical Ltd; equipped with a monochromatized Al K $\alpha$ X-ray source), respectively. The UV-Vis absorption and photoluminescence spectra were obtained using a UV-2450 spectrophotometer (Shimadzu) and an LS-55 fluorescence spectrometer (PerkinElmer), respectively.

\section{Cell imaging}

The HeLa cells (human epithelial carcinoma cell line; American Type Culture Collection) were cultured in DMEM (Life Technologies) supplemented with $10 \%$ fetal bovine serum and $1 \%$ penicillin-streptomycin, at $37{ }^{\circ} \mathrm{C}$ in a humidified atmosphere containing $5 \% \mathrm{CO}_{2}$ and $95 \%$ air. Before confocal imaging, the cells were incubated for $1-3 \mathrm{~h}$ with pTh-Pdots originally stocked in DMF $\left(5 \mu \mathrm{g} \mathrm{ml} \mathrm{m}^{-1}\right)$, pTh-Pdots originally stocked in water $\left(50 \mu \mathrm{g} \mathrm{ml} \mathrm{m}^{-1}\right.$ ), or LysoTracker Red DND-99 (100 nM; Life Technologies). In some experiments, the cells were transfected with mRFP-Rab5 (Addgene) using Lipofectamine3000 (Life Technologies), 1-2 days before incubation with pTh-Pdots. Confocal images were taken using a LSM710 confocal laserscanning microscope (Carl Zeiss, Germany) upon excitation at $405 \mathrm{~nm}, 488 \mathrm{~nm}$, or $580 \mathrm{~nm}$.

\section{Antibacterial studies}

All bacterial strains were from the American Type Culture Collection. Optical density at $600 \mathrm{~nm}$ (OD 600) of the bacterial suspensions was measured using a spectrophotometer (SpectraMax M5; Molecular Devices). pTh-Pdots or/and $\mathrm{H}_{2} \mathrm{O}_{2}$ (Sigma Aldrich) were added to $2 \mathrm{ml}$ of bacterial suspensions in Luria Broth (LB) solution ( 0.01 OD 600 corresponding to $1 \times 10^{6}$ cells per $\mathrm{ml}$ ), to attain a final concentration of $0-100 \mu \mathrm{g} \mathrm{m} \mathrm{m}^{-1}$ and $0-0.75 \mathrm{mM}$, respectively. This was followed by incubation at $37{ }^{\circ} \mathrm{C}$ for 8 or $18 \mathrm{~h}$, with $\sim 200 \mathrm{rpm}$ constant shaking. The OD change after the incubation was used to assess the inhibitory effect of pTh-Pdots or antibiotics on bacterial growth. The bactericidal activity of pTh-Pdots was examined using a fluorescence viability kit (Live/ Dead BacLight bacterial viability kit, Life Technologies) and FESEM. Before these imaging experiments, overnight grown E. coli cultures (OD $600=1.0$, corresponding to $1 \times 10^{9}$ cells per $\mathrm{ml}$ ) were centrifuged at $6000 \mathrm{rpm}$, followed by suspension in HBSS (Hank's balanced salt solution, Life Technologies) and $2 \mathrm{~h}$ incubation with or without $100 \mu \mathrm{g} \mathrm{ml}^{-1}$ pTh-Pdots. Subsequently, the suspension was centrifuged at $6000 \mathrm{rpm}$, washed thrice with PBS, and re-suspended in PBS. For FESEM, sample preparation follows the previously reported protocol. ${ }^{11}$ In brief, the bacteria were fixed by $2.5 \%$ glutaraldehyde (primary fixative) in $0.1 \mathrm{M}$ phosphate buffer $(\mathrm{PB})$ for $1 \mathrm{~h}$. The cells were then washed 3 times with $\mathrm{PB}$, followed by incubation in $1 \%$ osmium tetroxide (secondary fixative) for $1 \mathrm{~h}$. After washing 3 times with PB, the cells were subsequently dehydrated in ethanol with increasing concentrations $(25,50,75,90$, and $100 \%)$. Finally, the cells were re-suspended in the mixture of hexamethyldisilazane and ethanol (1:1) for $15 \mathrm{~min}$, followed by centrifugation at $2000 \times g$. The pellet was collected and allowed to dry overnight at room temperature.

\section{Results and discussion}

\section{Synthesis and characterizations}

As schematically illustrated in Scheme 1, polythiophene (pTh) is firstly electropolymerized from 2,2'-bithiophene (BTh) monomers in ionic liquid $\mathrm{BMIMBF}_{4}$, forming a thin film on the electrode surface. $\mathrm{BMIMBF}_{4}$ was used because of its wide electrochemical working window, excellent ionic conductivity and low volatility. ${ }^{12}$ As observed by field-effect scanning electron microscopy (FESEM), the pTh film consists of micro-granules whose size increases with polymerization time (Fig. S1 in ESI $\dagger$ ). Subsequently, pTh-Pdots are readily exfoliated from the pTh film by ultrasonication in anhydrous tetrahydrofuran (THF), yielding a yellowish homogenous suspension. Afterwards THF is completely extracted using rotary evaporation and the dried pTh-Pdots are re-suspended in dimethylformamide (DMF) or deionized water.

High-resolution transmission electron microscopy (HRTEM) reveals that the average diameter of pTh-Pdots is $2.37 \pm 0.47 \mathrm{~nm}$ $(n=200)$ (Fig. 1a and b). Atomic force microscopy (AFM) shows that the average thickness of pTh-Pdots is $3.83 \mathrm{~nm} \pm 0.73 \mathrm{~nm}$

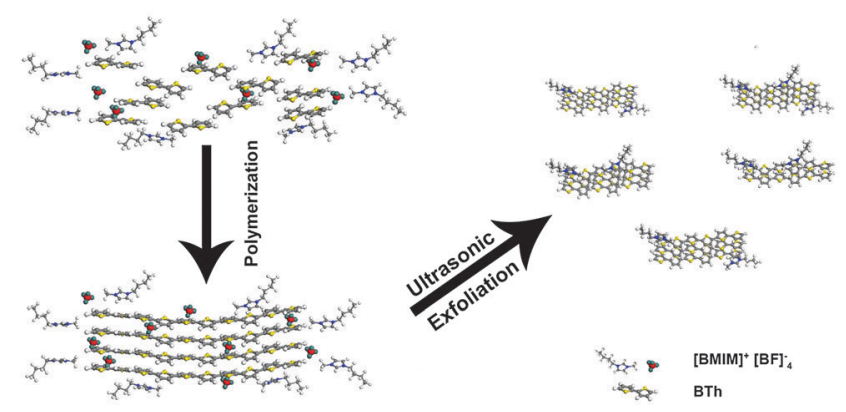

Scheme 1 Schematic illustration of pTh-Pdot synthesis. 

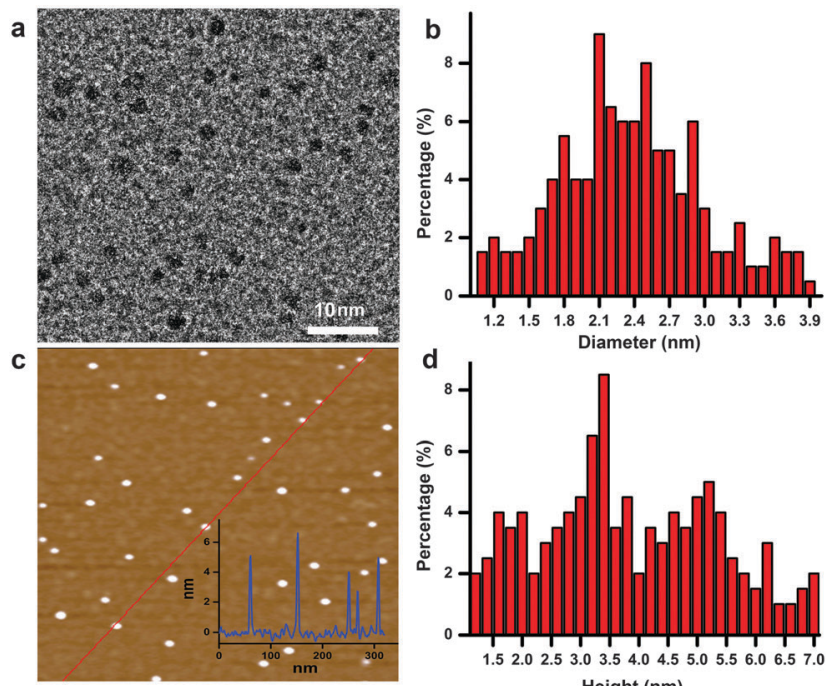

Fig. 1 (a) High-resolution TEM image of pTh-Pdots. (b) Diameter distribution $(n=200$ ). (c) AFM image of pTh-Pdots. Inset shows the height profile along the indicated line. (d) Height distribution $(n=200)$.

$(n=200)$, corresponding to $\sim 3$ layers of pTh (Fig. 1c and d). ${ }^{13,14}$ The Fourier transform infrared spectra (FTIR) of pTh-Pdots exhibit the stretching peaks for the thiophene ring $\left(1385 \mathrm{~cm}^{-1}\right)$, $\mathrm{C}-\mathrm{H}\left(1124 \mathrm{~cm}^{-1}\right), \mathrm{C}-\mathrm{O}\left(1084 \mathrm{~cm}^{-1}\right)$ and $\mathrm{C}-\mathrm{S}\left(697 \mathrm{~cm}^{-1}\right)$, which are characteristic of pTh (Fig. S2a in ESI $\dagger$ ) ${ }^{15,16}$ The triplet peaks of C-H asymmetric and symmetric vibration (at 2962, 2921, and $2852 \mathrm{~cm}^{-1}$ ) originate from the alkyl groups of $\mathrm{BMIM}^{+}$molecules adsorbed onto Pdots. ${ }^{17,18}$ Also characteristic of pTh, X-ray photoelectron spectroscopy (XPS) shows that the high-resolution $\mathrm{C}$ 1s spectrum can be deconvoluted into $\mathrm{sp}^{2}(\mathrm{C}=\mathrm{C}), \mathrm{sp}^{3}(\mathrm{C}-\mathrm{C}), \mathrm{C}-\mathrm{S}$ peaks; and the high-resolution $\mathrm{S} 2 \mathrm{p}$ spectrum can be split into $\mathrm{S} 2 \mathrm{p}_{3 / 2}$ and $\mathrm{S} 2 \mathrm{p}_{1 / 2}$ peaks (Fig. S2b and c in ESI $\dagger$ ). ${ }^{19,20}$ The $\mathrm{N}-\mathrm{C}$ and $\mathrm{N}=\mathrm{C}$ peaks in the high-resolution $\mathrm{N} 1 \mathrm{~s}$ spectrum originate from the imidazole moiety of $\mathrm{BMIM}^{+}$(Fig. S2d in ESI $\dagger$ ). ${ }^{21,22}$ Presumably, $\mathrm{BMIM}^{+}$facilitates the exfoliation and dispersion of Pdots through pi-pi interaction with $\mathrm{pTh} .{ }^{23}$ We found that compared to other ionic liquids such as 1-butyl-3-methylimidazolium methyl sulphate $\left(\mathrm{BMIMMeSO}_{4}\right)$ and tetra ethyl ammonium chloride (TEAC), BMIMBF ${ }_{4}$ offers the highest yield of pTh-Pdots.

Due to its amphiphilic properties, $\mathrm{BMIM}^{+}$assists pTh-Pdots to disperse well in both water and organic solvents (no obvious aggregation for months) (Fig. S3, ESI, $\dagger$ inset). ${ }^{23,24}$ Interestingly, pTh-Pdots in DMF appear to be green while they fluoresce blue in water under $365 \mathrm{~nm} \mathrm{UV}$ illumination (Fig. 2a, inset). It suggests that pTh-Pdots have negative solvatochromism, i.e., a blue shift in PL emission with increasing solvent polarity. Both pTh-Pdot suspensions can efficiently absorb UV light (Fig. 2a). The excitation spectra for both suspensions exhibit two peaks resulting from $\pi$ to $\pi^{*}$ and $\sigma$ to $\pi^{*}$ transitions (Fig. S3 in ESI $\dagger$ ). ${ }^{25}$ The maximum emission peaks for green pTh-Pdots in DMF and blue pTh-Pdots in water are achieved at $\sim 530 \mathrm{~nm}$ (excited by $440 \mathrm{~nm}$ ) and $\sim 435 \mathrm{~nm}$ (excited by $340 \mathrm{~nm}$ ), respectively (Fig. $2 \mathrm{~b}$ and c). In comparison, the 2,2'-bithiophene monomer at the concentration (dissolved in DMF; not soluble in water) does not
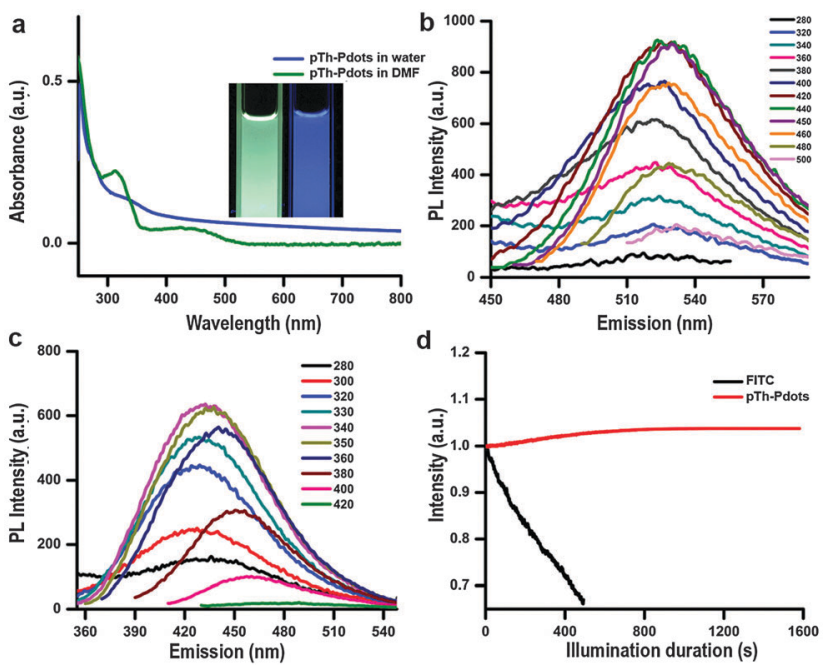

Fig. 2 (a) UV-Vis spectra of pTh-Pdots in DMF and pTh-Pdots in water. Inset shows the optical images under UV illumination at $365 \mathrm{~nm}$. (b) PL emission spectra of pTh-Pdots in DMF at different excitation wavelengths. (c) PL emission spectra of pTh-Pdots in water, (d) photo-bleaching of FITC dye molecules and $\mathrm{pTh}$-Pdots under confocal imaging.

exhibit any apparent light absorption or PL emission. PL quantum yields (QY) for the green pTh-Pdots in DMF and blue pTh-Pdots in water are calculated to be $44 \%$ (using R6G as reference) and $10 \%$ (using quinine sulfate as reference) respectively.

Negative solvatochromism of pTh-Pdots is presumably due to intra-molecular conformational changes in the conjugated backbone in the solvents of different polarities. ${ }^{26}$ As shown in Fig. S4 (ESI $\dagger$ ), with increasing solvent polarity (DMF, propanol, ethylene glycol, water) the emission of pTh-Pdots is blueshifted. Based on the observation that approximately the emission peak linearly scales with the relative polarity of the solvent, our pTh-Pdot may be used as a sensor to report solvent polarity. Consistently, adding $90 \%$ water to pTh-Pdot suspension in DMF changes its color from green to blue while with the addition of $50 \%$ water the pTh-Pdots can show both blue (excited at $340 \mathrm{~nm}$ ) and green (excited at $440 \mathrm{~nm}$ ) emission (Fig. S5 in ESI $\dagger$ ).

The use of organic fluorophores (e.g., FITC) for bioimaging is often plagued by their poor photo-stability, i.e., their fluorescence is quickly bleached under confocal laser illumination (Fig. 2d). In contrast, no obvious photo-bleaching of pTh-Pdots is observed under confocal imaging. Photobleaching of organic fluorophores is due to cleavage of covalent bonds or reactions with surrounding molecules or radicals. The high photo-stability of pTh-Pdots suggests that they are highly photochemically stable. Moreover, even at concentrations (e.g., $100 \mu \mathrm{g} \mathrm{ml}^{-1}$ ) much higher than needed for bioimaging (typically a few $\mu \mathrm{g} \mathrm{ml}^{-1}$ ), pTh-Pdots do not exert apparent cytotoxic effects on animal cells (Fig. S6 in ESI $\dagger$ ). Taken together, the high brightness, excellent photostability, good biocompatibility, and small size of pTh-Pdots are desirable properties for bioimaging.

\section{pTh-Pdots for cellular imaging}

As a proof-of-concept demonstration, we herein use pTh-Pdots for cellular imaging of HeLa cells. Although green pTh-Pdots 
stocked in DMF turn into blue after being diluted into bulk aqueous medium (Fig. S5 in ESI $\dagger$ ), they, as compared with blue pTh-Pdots stocked in water, can be more easily uptaken by the cells (compare Fig. 3 and Fig. S7 in the ESI $\dagger$ ). This is likely because the residual DMF molecules attached on pTh-Pdots facilitate the cell uptake. ${ }^{27}$ Therefore, in the following bioimaging experiments, we used pTh-Pdots originally stocked in DMF (i.e., DMF decorated pTh-Pdots). As shown in Fig. 3, after $1 \mathrm{~h}$ incubation with pTh-Pdots $\left(5 \mu \mathrm{g} \mathrm{ml}{ }^{-1}\right)$, pTh-Pdots are well taken up into the cells and appear blue. Apparently, these pTh-Pdots are segregated in the early endosomes after being endocytosed because they are largely co-localized with the early endosome marker mRFP-Rab5. Interestingly, after $3 \mathrm{~h}$ incubation, in addition to blue pTh-Pdots in early endosomes, green pThPdots also appear inside the cells. Evidently, these green pTh-Pdots stain lysosomes (but not early endosomes) because they are essentially co-localized with the lysosome marker LysoTracker Red (Fig. 3). These experiments demonstrate that pTh-Pdots can be employed to specifically label two distinct

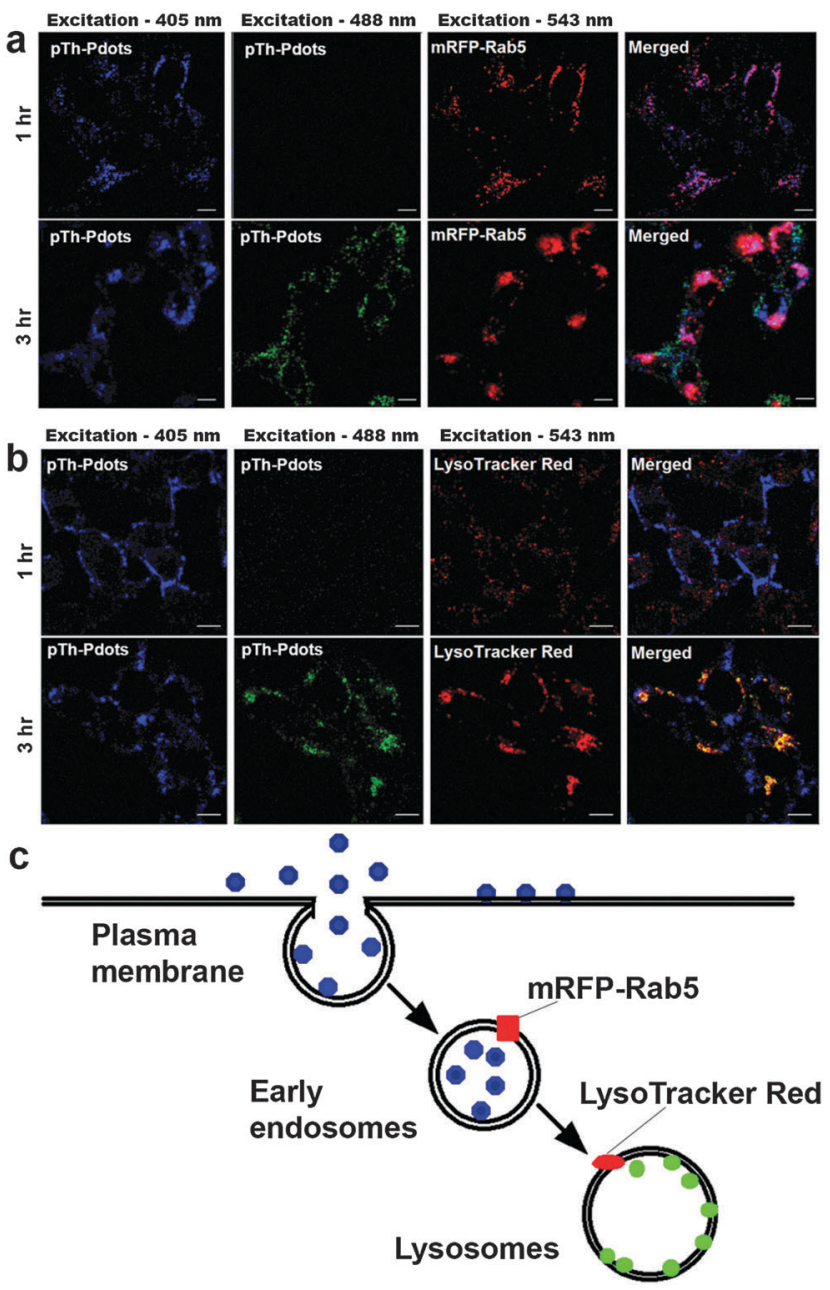

Fig. 3 Confocal imaging of HeLa cells incubated with pTh-Pdots (DMF stock diluted in cell medium to $5 \mu \mathrm{g} \mathrm{ml}^{-1}$ ) for 1 and $3 \mathrm{~h}$, respectively. (a) Co-localization with the early endosome marker (mRFP-Rab5). (b) Co-localization with the lysosome marker (LysoTracker red). (c) Schematic for the endocytosis uptake of pTh-Pdots. compartments (early endosomes and lysosomes) residing in the early and late endocytic pathways, respectively. As shown in Fig. S8 (ESI $\dagger$ ), after $6 \mathrm{~h}$ most of the pTh-Pdots move towards lysosomes and after $24 \mathrm{~h}$ all of them reach lysosomes. Taken together, pTh-Pdots migrate from early endosomes to lysosomes over time.

We conceive that owing to their polarity-sensitive PL properties pTh-Pdots fluoresce blue in the aqueous endosome lumen whereas they turn into green in lysosome because of being inserted into a hydrophobic lysosomal membrane. As discussed earlier, although polythiophene (pTh) is highly hydrophobic, pTh-Pdots can well-disperse in aqueous solution because of the attached $\mathrm{BMIM}^{+}$moieties. It is possible that $\mathrm{BMIM}^{+}$moieties are removed or destroyed while going through the early endocytic pathway or inside lysosomes, which are highly degradative because of the presence of various enzymes. As a consequence, the hydrophobic pTh-Pdots preferably intercalate into the lysosomal membrane. To directly support this hypothesis, we show that green pTh-Pdots originally stocked in DMF turn blue on being added to bulk water (Fig. S5 in ESI $\dagger$ ) whereas pTh-Pdots encapsulated by the amphiphilic polymer Pluronic F-127 retain green fluorescence in water (Fig. S9 in ESI $\dagger$ ). This confirms that pTh-Pdots are green fluorescent in a hydrophobic environment. We further show that pTh-Pdots incorporated into the lipid bilayers of artificial liposomes are green (Fig. S9 in $\mathrm{ESI} \dagger)$.

\section{pTh-Pdots as antibacterial agents}

With certain thiophene derivatives being known as good antibacterial agents, ${ }^{28,29}$ we speculate that pTh-Pdots can be used as antibacterial agents. Indeed, it is found that pTh-Pdots are very effective against both Gram-negative $[\mathrm{G}(-) ; E$. coli and $P$. aeruginosa] and Gram-positive $[\mathrm{G}(+) ; S$. aureus $]$ bacteria, with low minimum inhibitory concentrations (MIC, lowest concentration to cause appreciable inhibition of bacterial growth). For these bacterial strains, the potency of pTh-Pdots is comparable to a commonly used broad-spectrum antibiotic (kanamycin). Dose dependent studies show that pTh-Pdots are most effective against $E$. coli with MIC $\sim 45 \mu \mathrm{g} \mathrm{ml}^{-1}$, followed by $S$. aureus $\left(\mathrm{MIC} \sim 60 \mu \mathrm{g} \mathrm{ml}^{-1}\right.$ ) and P. aeruginosa $\left(\mathrm{MIC} \sim 100 \mu \mathrm{g} \mathrm{ml}^{-1}\right.$ ) (Fig. $4 \mathrm{a}$ and $\mathrm{b}$ and Fig. S10 in ESI $\dagger$ ). Unsurprisingly, pTh-Pdots are more potent to $G(-)$ bacteria than $G(+)$ bacteria which are protected by a thick peptidoglycan surface layer.

Some pathogenic $E$. coli strains can be life threatening and resistant to antibiotics. As shown in Fig. 4c, pTh-Pdots can efficiently kill ampicillin-resistant $E$. coli. On the other hand, pTh-Pdots are highly biocompatible to mammalian cells (primary rat fibroblast cells in Fig. 4d; HeLa cells in Fig. S6 in ESI $\dagger$ ). The antibacterial capability of pTh-Pdots does not originate from $\mathrm{BMIM}^{+}$moieties attached on their surface because $\mathrm{BMIMBF}_{4}$ ionic liquid even at a high concentration of $0.5 \mathrm{mg} \mathrm{ml}^{-1}$ is incapable of causing bacterial death (Fig. S11 in ESI $\dagger$ ).

Also interestingly, we discover that the pTh-Pdot is an excellent peroxidase mimic, which converts $\mathrm{H}_{2} \mathrm{O}_{2}$ into hydroxyl radicals $\left({ }^{\circ} \mathrm{OH}\right)$ (Fig. S12 in ESI $\dagger$ ). Graphene quantum dots (GQDs) have also been found to show peroxidase activities. ${ }^{30,31}$ 

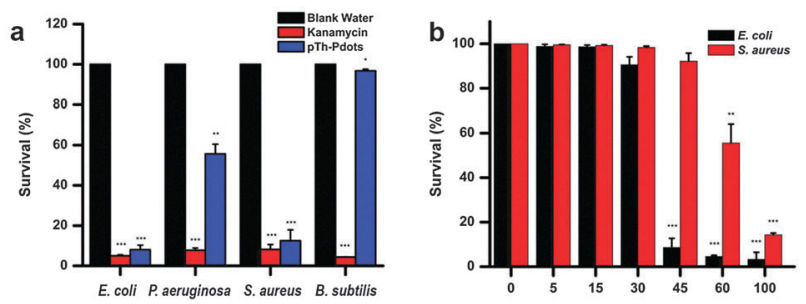

C

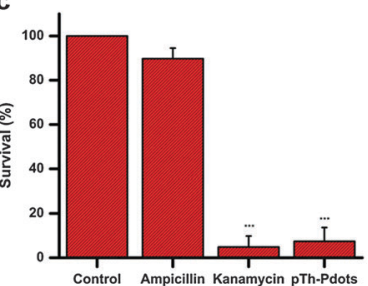

d Concentration of $\mathrm{pTh}$-Pdots $(\mu \mathrm{g} / \mathrm{ml})$
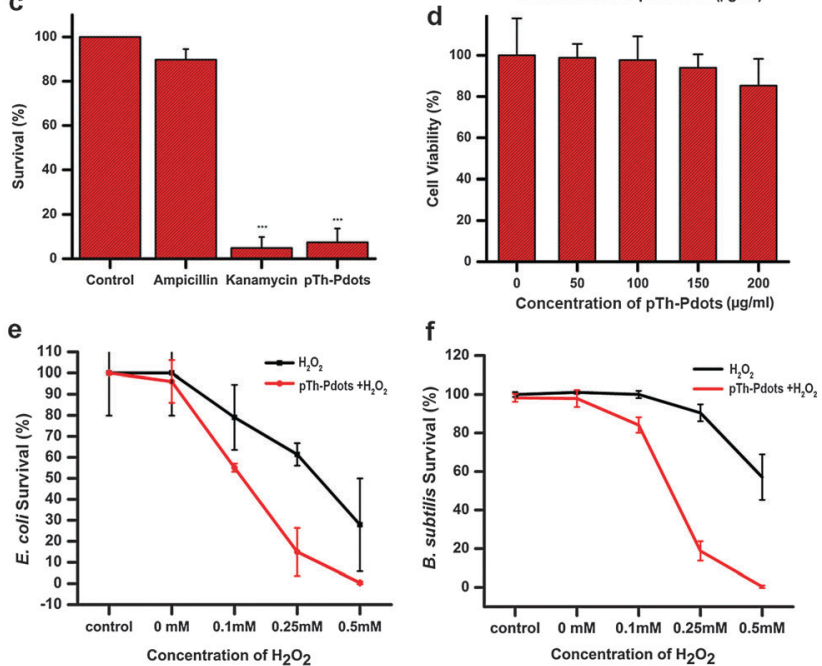

Fig. 4 Antibacterial activity of pTh-Pdots. (a) Viability test against $\mathrm{G}(+)$ and $\mathrm{G}(-)$ bacteria with $100 \mu \mathrm{g} \mathrm{ml}^{-1}$ pTh-Pdots. (b) Dose dependent effect of pTh-Pdots against $\mathrm{G}(+)$ and $\mathrm{G}(-)$ bacteria. (c) Viability test of ampicillinresistant $E$. coli treated with ampicillin, kanamycin and pTh-Pdots (all at $100 \mu \mathrm{g} \mathrm{ml}{ }^{-1}$ ). (d) MTT assay of rat fibroblasts after overnight incubation with $\mathrm{pTh}$-Pdots at various concentrations. (e and f) Viability tests of $E$. coli (e) and $B$. subtilis (f) treated with $\mathrm{H}_{2} \mathrm{O}_{2}$ at different concentrations with or without pTh-Pdots $\left(10 \mu \mathrm{g} \mathrm{ml}^{-1}\right)$.

Taking advantage of this, they have been used to improve the antibacterial performance of $\mathrm{H}_{2} \mathrm{O}_{2}$ for wound disinfection. ${ }^{32}$ We found that pTh-Pdots possess much higher peroxidase activity
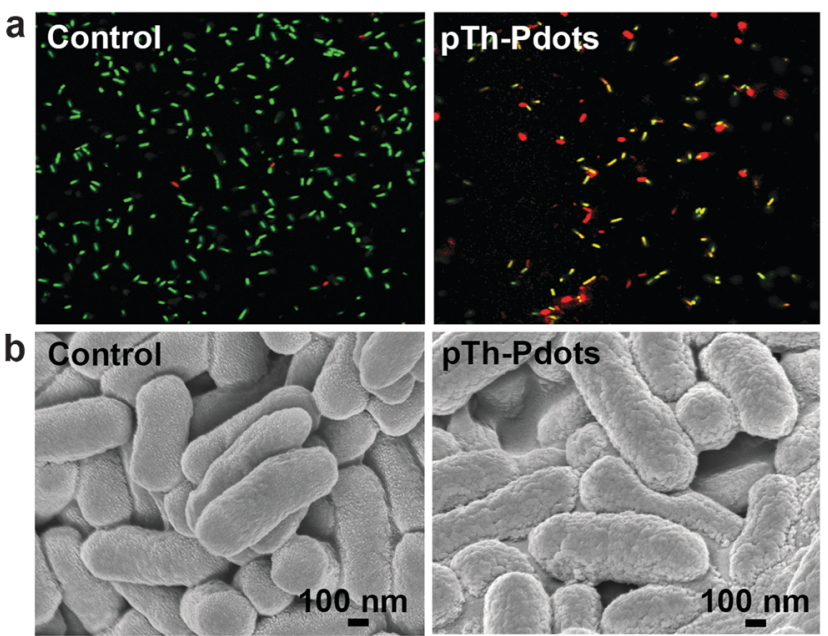

Fig. 5 Fluorescence images with live/dead dye staining (a) and FESEM images of $E$. coli cells (b) without (left column) or with (right column) incubation of pTh-Pdots $(100 \mu \mathrm{g})$ for $2 \mathrm{~h}$. than GQDs (Fig. S12 in ESI $\dagger$ ). Although pTh-Pdot itself cannot kill Gram-positive B. subtilis (Fig. 4a), the synergistic effect between pTh-Pdots and $\mathrm{H}_{2} \mathrm{O}_{2}$ is potent (Fig. 4f). pTh-Pdots can greatly enhance the potency of $\mathrm{H}_{2} \mathrm{O}_{2}$ in killing both Gram-negative (Fig. 4e) and Gram-positive strains (Fig. 4f).

We speculate that the antimicrobial activity of the positively charged pTh-Pdots (zeta potential $\zeta=21.2 \mathrm{mV}$ ) may be realized by disrupting the negatively charged bacterial membrane through electrostatic interaction. Indeed as shown in Fig. 5a, in the presence of a viability testing dye mixture (SYTO 9/propidium iodide), live bacteria appear green due to SYTO 9 staining whereas dying or dead bacteria after pTh-Pdot treatment appear yellow or red due to the uptake of red propidium iodide through a compromised cell membrane. Consistently, field-effect scanning electron microscopy (FESEM) also shows that the bacterial membrane becomes crumpled after pTh-Pdot treatment (Fig. 5b).

\section{Conclusion}

In summary, we have synthesized a new fluorescent polymer dot derived from non-fluorescent polythiophene (pTh-Pdots) using a simple and high yield procedure. These pTh-Pdots are bright, stable, biocompatible and small, and therefore promising for bioimaging applications. Exploiting its negative solvatochromism properties, here we demonstrate the use of pTh-Pdots in live cells to label and differentiate early endosomes and lysosomes along the endocytic pathway. We further demonstrate that, making use of their ability to disrupt the bacterial membrane and mimic peroxidase activity, pTh-Pdots can serve as potent antibacterial agents against both Gram-negative and Gram-positive bacteria, even drug-resistant strains. Different types of Pdots may be similarly synthesized simply by changing the polymeric precursors (fluorescent or non-fluorescent, conducting or semiconducting). Distinct to other reported polymer dots, which are made by condensing the long-chain fluorescent semiconducting polymers, the Pdots presented here are synthesized from the bottom up and are much smaller. Such a new class of zerodimensional nanomaterials, which may be viewed as heteroatomdoped carbon nanoparticles and fluoresce due to quantum confinement induced bandgap opening, ${ }^{33,34}$ shall find a wide range of novel applications.

\section{Acknowledgements}

This work was supported by the Ministry of Education of Singapore under the AcRF Tier 2 grant (MOE2014-T2-1-003), AcRF Tier 1 grant (M4011559.120, RG133/15) and the Nanyang Technological University start-up grant (NTU-SUG: M4081627.120).

\section{Notes and references}

1 C. Wu and D. T. Chiu, Angew. Chem., Int. Ed. Engl., 2013, 52, 3086-3109.

2 Z. Tian, J. Yu, C. Wu, C. Szymanski and J. McNeill, Nanoscale, 2010, 2, 1999-2011. 
3 R. I. Dmitriev, S. M. Borisov, H. Dussmann, S. Sun, B. J. Muller, J. Prehn, V. P. Baklaushev, I. Klimant and D. B. Papkovsky, ACS Nano, 2015, 9, 5275-5288.

4 X. B. Zhou, H. Liang, P. F. Jiang, K. Y. Zhang, S. J. Liu, T. S. Yang, Q. Zhao, L. J. Yang, W. Lv, Q. Yu and W. Huang, Adv. Sci., 2016, 3, 1500155.

5 Y. R. Zhang, L. Pang, C. Ma, Q. Tu, R. Zhang, E. Saeed, A. Mahmoud and J. Y. Wang, Anal. Chem., 2014, 86, 3092-3099.

6 D. Zhang, M. Wu, Y. Y. Zeng, N. S. Liao, Z. X. Cai, G. Liu, X. L. Liu and J. F. Liu, J. Mater. Chem. B, 2016, 4, 589-599.

7 J. Pecher and S. Mecking, Chem. Rev., 2010, 110, 6260-6279.

8 C. Wu, C. Szymanski, Z. Cain and J. McNeill, J. Am. Chem. Soc., 2007, 129, 12904-12905.

9 K. Landfester, R. Montenegro, U. Scherf, R. Guntner, U. Asawapirom, S. Patil, D. Neher and T. Kietzke, Adv. Mater., 2002, 14, 651-655.

10 K. P. Prasad, Y. Chen, M. A. Sk, A. Than, Y. Wang, H. D. Sun, K. H. Lim, X. C. Dong and P. Chen, Mater. Horiz., 2014, 1, 529-534.

11 E. R. Fischer, B. T. Hansen, V. Nair, F. H. Hoyt and D. W. Dorward, Curr. Protoc. Microbiol., John Wiley \& Sons, Inc., 2012, vol. 25, pp. 2B.2.1-2B.2.47.

12 Y. H. Pang, X. Y. Li, G. Y. Shi, F. Wang and L. T. Jin, Thin Solid Films, 2008, 516, 6512-6516.

13 J. H. Liu, I. A. Mikhailov, I. Osaka, A. E. Masunov, R. D. McCullough and L. Zhai, Polymer, 2011, 52, 2302-2309.

14 G. Adam, A. Pivrikas, A. M. Ramil, S. Tadesse, T. Yohannes, N. S. Sariciftci and D. A. M. Egbe, J. Mater. Chem., 2011, 21, 2594-2600.

15 B. Massoumi, N. Alipour, S. Fathalipour and M. Jaymand, High Perform. Polym., 2015, 27, 161-170.

16 D. Sun, L. Jin, Y. Chen, J. R. Zhang and J. J. Zhu, ChemPlusChem, 2013, 78, 227-234.

17 A. Ananthanarayanan, X. W. Wang, P. Routh, B. Sana, S. Lim, D. H. Kim, K. H. Lim, J. Li and P. Chen, Adv. Funct. Mater., 2014, 24, 3021-3026.

18 S. Cha, M. Ao, W. Sung, B. Moon, B. Ahlstrom, P. Johansson, Y. Ouchi and D. Kim, Phys. Chem. Chem. Phys., 2014, 16, 9591-9601.

19 E. L. Ratcliff, R. C. Bakus, G. C. Welch, T. S. van der Poll, A. Garcia, S. R. Cowan, B. A. MacLeod, D. S. Ginley,
G. C. Bazan and D. C. Olson, J. Mater. Chem. C, 2013, 1, 6223-6234.

20 E. Z. Kurmaev, S. N. Shamin, V. R. Galakhov, A. Moewes, T. Otsuka, S. Koizume, K. Endo, H. E. Katz, M. Bach, M. Neumann, D. L. Ederer and M. Iwami, Phys. Rev. B: Condens. Matter Mater. Phys., 2001, 64, 452111.

21 H. J. Kim, I. S. Bae, S. J. Cho, J. H. Boo, B. C. Lee, J. Heo, I. Chung and B. Hong, Nanoscale Res. Lett., 2012, 7, 1-7.

22 O. Hofft, S. Bahr, M. Himmerlich, S. Krischok, J. A. Schaefer and V. Kempter, Langmuir, 2006, 22, 7120-7123.

23 T. L. Greaves and C. J. Drummond, Chem. Soc. Rev., 2008, 37, 1709-1726.

24 M. L. Wang, Y. Q. Gao, J. J. Zhang and J. W. Zhao, Electrochim. Acta, 2015, 155, 236-243.

25 A. Baheti, C. P. Lee, K. R. Thomas and K. C. Ho, Phys. Chem. Chem. Phys., 2011, 13, 17210-17221.

26 M. Leclerc, G. Dufresne, P. Blondin, J. Bouchard, M. Belletete and G. Durocher, Synth. Met., 2001, 119, 45-48.

27 M. Katz and B. J. Poulsen, in Concepts in Biochemical Pharmacology: Part 1, ed. B. B. Brodie, J. R. Gillette and H. S. Ackerman, Springer Berlin Heidelberg, Berlin, Heidelberg, 1971, vol. XXVIII, pp. 103-174.

28 R. Wilson, P. Kumar, V. Parashar, C. Vilchèze, R. VeyronChurlet, J. S. Freundlich, S. W. Barnes, J. R. Walker, M. J. Szymonifka, E. Marchiano, S. Shenai, R. Colangeli, W. R. Jacobs Jr, M. B. Neiditch, L. Kremer and D. Alland, Nat. Chem. Biol., 2013, 9, 499-506.

29 M. L. Cerrada, A. Munoz-Bonilla and M. Fernandez-Garcia, Polymeric Materials with Antimicrobial Activity: From Synthesis to Applications, The Royal Society of Chemistry, 2014, pp. 387-405.

30 N. Li, A. Than, X. Wang, S. Xu, L. Sun, H. Duan, C. Xu and P. Chen, ACS Nano, 2016, 10, 3622-3629.

31 H. Sun, A. Zhao, N. Gao, K. Li, J. Ren and X. Qu, Angew. Chem., 2015, 54, 7176-7180.

32 H. J. Sun, N. Gao, K. Dong, J. S. Ren and X. G. Qu, ACS Nano, 2014, 8, 6202-6210.

33 X. T. Zheng, A. Ananthanarayanan, K. Q. Luo and P. Chen, Small, 2015, 11, 1620-1636.

34 D. V. Lap, D. Grebner and S. Rentsch, J. Phys. Chem. A, 1997, 101, 107-112. 